\title{
Treewidth of the Kneser Graph and the Erdös-Ko-Rado Theorem
}

\author{
Daniel J. Harvey * \\ Department of Mathematics and Statistics \\ The University of Melbourne \\ Melbourne, Australia \\ d.harvey@pgrad.unimelb.edu .au
}

\author{
David R. Wood ${ }^{\dagger}$ \\ School of Mathematical Sciences \\ Monash University \\ Melbourne, Australia \\ david.wood@monash.edu
}

Submitted: Dec 17, 2013; Accepted: Feb 20, 2014; Published: Feb 28, 2014

Mathematics Subject Classifications: 05C75, 05D05

\begin{abstract}
Treewidth is an important and well-known graph parameter that measures the complexity of a graph. The Kneser graph $\operatorname{Kneser}(n, k)$ is the graph with vertex set $\left(\begin{array}{c}{[n]} \\ k\end{array}\right)$, such that two vertices are adjacent if they are disjoint. We determine, for large values of $n$ with respect to $k$, the exact treewidth of the Kneser graph. In the process of doing so, we also prove a strengthening of the Erdös-Ko-Rado Theorem (for large $n$ with respect to $k$ ) when a number of disjoint pairs of $k$-sets are allowed.
\end{abstract}

Keywords: graph theory; Kneser graph; treewidth; separators; Erdős-Ko-Rado

\section{Introduction}

A tree decomposition of a graph $G$ is a pair $\left(T,\left(B_{x} \subset V(G): x \in V(T)\right)\right)$ where $T$ is a tree and $\left(B_{x} \subseteq V(G): x \in V(T)\right)$ is a collection of sets, called bags, indexed by the nodes of $T$. The following properties must also hold:

- for each $v \in V(G)$, the nodes of $T$ that index the bags containing $v$ induce a nonempty connected subtree of $T$,

- for each $v w \in E(G)$, there exists some bag containing both $v$ and $w$.

* Supported by an Australian Postgraduate Award.

${ }^{\dagger}$ Supported by the Australian Research Council. 
The width of a tree decomposition is the size of the largest bag, minus 1 . The treewidth of a graph $G$, denoted $\operatorname{tw}(G)$, is the minimum width of a tree decomposition of $G$.

Treewidth is an important concept in modern graph theory. Treewidth was initially defined by Halin [6] (with different nomenclature to the modern standard) and then later by Robertson and Seymour [16], who used it in their famous series of papers proving the Graph Minor Theorem [15]. The treewidth of a graph essentially describes how "tree-like" it is, where lower treewidth implies a more "tree-like" structure. (A forest has treewidth at most 1, for example.) Treewidth is also of key interest in the field of algorithm design - for example, treewidth is a key parameter in fixed-parameter tractability [1].

Let $[n]=\{1, \ldots, n\}$. For any set $S \subseteq[n]$, a subset of $S$ of size $k$ is called a $k$-set, or occasionally a $k$-set in $S$. Let $\left(\begin{array}{l}S \\ k\end{array}\right)$ denote the set of all $k$-sets in $S$. We say two sets intersect when they have non-empty intersection.

The Kneser graph $\operatorname{Kneser}(n, k)$ is the graph with vertex set $\left(\begin{array}{c}{[n]} \\ k\end{array}\right)$, such that two vertices are adjacent if they are disjoint.

Kneser graphs were first investigated by Kneser [9]. The chromatic number of the graph $\operatorname{Kneser}(n, k)$ was shown to be $n-2 k+2$ by Lovász [11], as Kneser originally conjectured. This was an important proof due to the development of the topological methods involved. Many other proofs of this result have been found, for example consider [19], which gives a more combinatorial version. The Kneser graph is also of interest with regards to fractional chromatic number [17]. The famous Erdős-Ko-Rado Theorem [2] has a well-known relationship to the Kneser graph, as does the generalisation to crossintersecting families by Pyber [14]. We discuss these in more detail in Section 2, and shall use both of these results to prove the following two theorems about the treewidth of the Kneser graph.

Theorem 1. Let $G$ be a Kneser graph with $n \geqslant 4 k^{2}-4 k+3$ and $k \geqslant 3$. Then

$$
\operatorname{tw}(G)=\left(\begin{array}{c}
n-1 \\
k
\end{array}\right)-1 .
$$

This theorem is our main result, giving an exact answer for the treewidth of the Kneser graph when $n$ is sufficiently large. In order to prove this, we show that $\left(\begin{array}{c}n-1 \\ k\end{array}\right)-1$ is both an upper bound and lower bound on the treewidth. We construct a tree decomposition directly in Section 3 to prove an upper bound. In Section 4 we prove the lower bound by using the relationship between treewidth and separators. In Section 6, we further conjecture that Theorem 1 extends to lower values of $n$.

We also prove the following more precise result when $k=2$.

Theorem 2. Let $G$ be a Kneser graph with $k=2$. Then

$$
\operatorname{tw}(G)= \begin{cases}0 & \text { if } n \leqslant 3 \\
1 & \text { if } n=4 \\
4 & \text { if } n=5 \\
\left(\begin{array}{c}
n-1 \\
2
\end{array}\right)-1 & \text { if } n \geqslant 6 .\end{cases}
$$


The upper bounds for Theorem 2 are proved in Section 3, and the lower bounds in Section 5.

Finally, in the process of proving Theorem 1, we prove the following generalisation of the Erdös-Ko-Rado Theorem (Theorem 6 in Section 2), which says that if $n \geqslant 2 k$ and $H$

is a complete subgraph in the complement of $\operatorname{Kneser}(n, k)$ then $|H| \leqslant\left(\begin{array}{l}n-1 \\ k-1\end{array}\right)$. We prove the same bound for balanced complete multipartite graphs.

Theorem 3. Say $p \in\left[\frac{2}{3}, 1\right)$ and $n \geqslant \max \left(4 k^{2}-4 k+3, \frac{1}{1-p}\left(k^{2}-1\right)+2\right)$. If $H$ is a complete multipartite subgraph of the complement of $\operatorname{Kneser}(n, k)$ such that no colour class contains more than $p|H|$ vertices, then $|H| \leqslant\left(\begin{array}{l}n-1 \\ k-1\end{array}\right)$.

Note that similar, but incomparable, generalisations of the Erdös-Ko-Rado Theorem have recently been explored in $[5,4,18]$. Theorem 3 is proven in Section 4 , since it follows almost directly from our proof of the lower bound on the treewidth of a Kneser graph.

\section{Basic Definitions and Preliminaries}

From now on, we refer to the graph $\operatorname{Kneser}(n, k)$ as $G$, with $n$ and $k$ implicit.

Let $\Delta(H)$ be the maximum degree of a graph $H$ and $\delta(H)$ be the minimum degree of a graph $H$. Also let $\alpha(H)$ be the size of the largest independent set of $H$, where an independent set is a set of pairwise non-adjacent vertices. If $k=1$, then $G$ is the complete graph. If $n<2 k$ then $G$ contains no edges. If $n=2 k$ then $G$ is an induced matching. From now on, we shall assume that $n \geqslant 2 k+1$ and $k \geqslant 2$, since the treewidth is trivial in the other cases.

In order to prove a lower bound on the treewidth of the Kneser graph, we use a known result about the relationship between treewidth and separators.

Definition Given a constant $p \in\left[\frac{2}{3}, 1\right.$ ), a p-separator (of order $k$ ) is a set $X \subset V(G)$ such that $|X| \leqslant k$ and no component of $G-X$ contains more than $p|G-X|$ vertices.

Theorem 4. [16] For each $p \in\left[\frac{2}{3}, 1\right)$, every graph $G$ has a $p$-separator of order $\operatorname{tw}(G)+1$.

It can easily be shown that we can partition the components of $G-X$ into two parts, such that the components in a part contain, in total, at most $p|G-X|$ vertices. This gives the following lemma.

Lemma 5. Let $X$ be a p-separator. Then $V(G-X)$ can be partitioned into two parts $A$ and $B$, with no edge between $A$ and $B$, such that

- $(1-p)|G-X| \leqslant|A| \leqslant \frac{1}{2}|G-X|$,

- $\frac{1}{2}|G-X| \leqslant|B| \leqslant p|G-X|$.

We use a few important well known combinatorial results. 
Theorem 6 (Erdős-Ko-Rado $[2,7])$. Let $G$ be $\operatorname{Kneser}(n, k)$ for some $n \geqslant 2 k$. Then $\alpha(G)=\left(\begin{array}{c}n-1 \\ k-1\end{array}\right)$. If $n \geqslant 2 k+1$ and $\mathcal{A}$ is an independent set such that $|\mathcal{A}|=\left(\begin{array}{l}n-1 \\ k-1\end{array}\right)$, then $\mathcal{A}=\{v \mid i \in v\}$ for a fixed element $i \in[n]$.

The original Erdős-Ko-Rado Theorem defines $\mathcal{A}$ as a set of $k$-sets in [n], such that the $k$-sets of $\mathcal{A}$ pairwise intersect. Our formulation in terms of vertices in the Kneser graph is clearly equivalent. We will use Theorem 6 when determining an upper bound for $\operatorname{tw}(G)$.

The second major result is by Pyber [14]. Let $\mathcal{A}$ and $\mathcal{B}$ be sets of vertices of the Kneser graph $G$, such that for all $v \in \mathcal{A}$ and $w \in \mathcal{B}$ the pair $v w$ is not an edge. Then we say the pair $(\mathcal{A}, \mathcal{B})$ are cross-intersecting families.

Theorem 7 (Erdős-Ko-Rado for Cross-Intersecting Families [14, 13]). Let $n \geqslant 2 k$ and let $(\mathcal{A}, \mathcal{B})$ be cross-intersecting families in the Kneser graph $G$. Then $|\mathcal{A}||\mathcal{B}| \leqslant\left(\begin{array}{c}n-1 \\ k-1\end{array}\right)^{2}$. If $n \geqslant 2 k+1$ and $(\mathcal{A}, \mathcal{B})$ are cross-intersecting families such that $|\mathcal{A}||\mathcal{B}|=\left(\begin{array}{l}n-1 \\ k-1\end{array}\right)^{2}$, then $\mathcal{A}=\mathcal{B}=\{v \mid i \in v\}$ for a fixed element $i \in[n]$.

As with Theorem 6, the original formulation by Pyber of Theorem 7 is more general. We have given the result in an equivalent form that is sufficient for our requirements.

Let $X$ be a $\frac{2}{3}$-separator and $A, B$ the parts of the vertex partition of $G-X$ as in Lemma 5. Now for all $v \in A$ and $w \in B, v$ and $w$ are in different components and as such are non-adjacent. So $(A, B)$ are cross-intersecting families. We know $|A|=c|G-X|$ where $\frac{1}{3} \leqslant c \leqslant \frac{1}{2}$. By Theorem 7 , it follows that $c(1-c)|G-X|^{2} \leqslant\left(\begin{array}{c}n-1 \\ k-1\end{array}\right)^{2}$. It follows that $|G-X| \leqslant \frac{3}{\sqrt{2}}\left(\begin{array}{c}n-1 \\ k-1\end{array}\right)$. (We leave the precise calculation to the reader.) This gives a lower bound on $|X|$, and as such a lower bound on the treewidth (by Theorem 4). Hence $\operatorname{tw}(G) \geqslant\left(\begin{array}{l}n \\ k\end{array}\right)-\frac{3}{\sqrt{2}}\left(\begin{array}{l}n-1 \\ k-1\end{array}\right)-1$.

However, note that the parts $A$ and $B$ of $V(G-X)$ are vertex disjoint, but that the definition of a pair of cross-intersecting families does not require this. In fact, Theorem 7 shows that in the case where $|\mathcal{A}||\mathcal{B}|$ is maximised, $\mathcal{A}=\mathcal{B}$. We show we can do better than the above naïve lower bound on $\operatorname{tw}(G)$ when $\mathcal{A}$ and $\mathcal{B}$ are disjoint.

Before considering our final preliminary, we provide the following definitions. Consider all of the $a$-sets in [b]. Define the colexicographic or colex ordering on the $a$-sets as follows: if $x$ and $y$ are distinct $a$-sets, then $x<y$ when $\max (x-y)<\max (y-x)$. This is a strict total order. A set $X$ of $a$-sets in $[b]$ is first if $X$ consists of the first $|X| a$-sets in the colex ordering of all the $a$-sets in $[b]$.

Now consider the colex ordering of $a$-sets in [b]. All of the $a$-sets in [i] (where $i<b$ ) come before any $a$-set containing an element greater than or equal to $i+1$. To see this, note if $x$ is an $a$-set in $[i]$ and $y$ is an $a$-set with $j \in y$ such that $j \geqslant i+1$, then $\max (x-y) \leqslant \max (x) \leqslant i$, and $\max (y-x) \geqslant j \geqslant i+1$ since $j \in y-x$. We will use this when determining the make-up of first sets in Section 4.

Let $X$ be a set of $a$-sets in [b]. For $c \leqslant a$, the $c$-shadow of $X$ is the set $\{x:|x|=c$, and $\exists y \in X$ such that $x \subseteq y\}$. That is, the $c$-shadow contains all $c$-sets that are contained within $a$-sets of $X$. If $x$ is an $a$-set in [b], let the complement of $x$ be the $(b-a)$-set $y=[b]-x$. If $X$ is a set of $a$-sets on [b], then the complement of $X$ is $\bar{X}:=\{y: y$ is the complement of some $x \in X\}$. Note $|X|=|\bar{X}|$. 
Lemma 8 (A first set minimises the shadow [10, 8] (see [3] for a short proof)). Let $X$ be a set of a-sets on $[b], c \leqslant a$ and $S$ be the c-shadow of $X$. Suppose $|X|$ is fixed but $X$ is not. Then $|S|$ is minimised when $X$ is first.

This idea is also used by Pyber [14] and Matsumoto and Tokushige [13]. Intuitively, the shadow $S$ should be minimised whenever the $a$-sets of $X$ "overlap" as much as possible, so that each $c$-set in $S$ is a subset of as many $a$-sets as possible.

\section{$3 \quad$ Upper Bound for Treewidth}

This section proves the upper bounds on $\operatorname{tw}(G)$ in Theorems 1 and 2 .

In both Theorem 1 and 2, the upper bound is almost always $\left(\begin{array}{c}n-1 \\ k\end{array}\right)-1$. The only exceptions are the trivial cases (when $n \leqslant 2 k$ ), and the case when $k=2$ and $n=5$, which is the Petersen graph. The Petersen graph is well-known to have treewidth 4 ([12], for example). What follows is a general upper bound on the treewidth of any graph, which is sufficient to prove the remaining cases.

Lemma 9. If $H$ is any graph, then $\operatorname{tw}(H) \leqslant \max \{\Delta(H),|V(H)|-\alpha(H)-1\}$.

Proof. Let $\alpha:=\alpha(H)$. We shall construct a tree decomposition with underlying tree $T$, where $T$ is a star with $\alpha(H)$ leaves. Let $R$ be the bag indexed by the central node of $T$, and label the other bags $B_{1}, \ldots, B_{\alpha}$. Let $X:=\left\{x_{1}, \ldots x_{\alpha}\right\}$ be a maximum independent set in $H$. Let $R:=V(H)-X$ and $B_{i}:=N\left(x_{i}\right) \cup\left\{x_{i}\right\}$ for all $i \in\{1, \ldots, \alpha\}$. We now show this is a tree decomposition:

Any vertex not in $X$ is contained in $R$. Given the structure of the star, any induced subgraph containing the central node is connected. Alternatively, if a vertex is in $X$, then it appears only in bags indexed by leaves. However, since $X$ is an independent set, $x_{i} \in X$ appears only in $B_{i}$, not in any other bag $B_{j}$. A single node is obviously connected. If $v w$ is an edge of $H$, then at most one of $v$ and $w$ is in $X$. Say $v=x_{i} \in X$. Then $v, w$ both appear in the bag $B_{i}$. Otherwise neither vertex is in $X$, and both vertices appear in $R$.

So this is a tree decomposition. The size of $R$ is $|V(H)|-\alpha(H)$. The size of $B_{i}$ is the degree of $x_{i}$, plus one, which is at most $\Delta(H)+1$. From here our lemma is proven.

We now consider this result for the Kneser graph itself.

Lemma 10. If $G$ is a Kneser graph with $k \geqslant 2$ and $n \geqslant 2 k+1$, then $\operatorname{tw}(G) \leqslant\left(\begin{array}{c}n \\ k-1\end{array}\right)-1$.

Proof. By Lemma 9 and Theorem 6, and since $n \geqslant 2 k+1$,

$$
\operatorname{tw}(G) \leqslant \max \{\Delta(G),|V(G)|-\alpha(G)-1\}=\max \left\{\left(\begin{array}{c}
n-k \\
k
\end{array}\right),\left(\begin{array}{l}
n \\
k
\end{array}\right)-\left(\begin{array}{c}
n-1 \\
k-1
\end{array}\right)-1\right\} .
$$

Since $k \geqslant 2, \operatorname{tw}(G) \leqslant\left(\begin{array}{c}n-1 \\ k\end{array}\right)-1$, as required. 


\section{Separators in the Kneser Graph}

To complete the proof of Theorem 1, it is sufficient to prove a lower bound on the treewidth. The following lemma, together with Theorem 4, provides this. It is the heart of the proof of Theorem 3 .

Lemma 11. Let $X$ be a p-separator of the Kneser graph $G$. If $n \geqslant \max \left(4 k^{2}-4 k+\right.$ $\left.3, \frac{1}{1-p}\left(k^{2}-1\right)+2\right)$, then $|X| \geqslant\left(\begin{array}{c}n-1 \\ k\end{array}\right)$.

Proof. Assume, for the sake of a contradiction, that $|X|<\left(\begin{array}{c}n-1 \\ k\end{array}\right)$. Then $|G-X|>\left(\begin{array}{c}n-1 \\ k-1\end{array}\right)$. By Lemma $5, G-X$ has two parts $A$ and $B$ such that $(1-p)|G-X| \leqslant|A| \leqslant \frac{1}{2}|G-X|$ and $\frac{1}{2}|G-X| \leqslant|B| \leqslant p|G-X|$ and no edge has an endpoint in both $A$ and $B$.

For a given element $i \in[n]$, let $A_{i}:=\{v \in A: i \in v\}$. Also define $A_{-i}:=\{v \in A: i \notin$ $v$ \}. So $A_{i}$ and $A_{-i}$ partition the set $A$, for any choice of $i$. Define analogous sets for $B$. Claim 1. There exists some $i$ such that $\left|B_{i}\right| \geqslant \frac{1}{k}|B|$.

Proof. Since $|A| \geqslant(1-p)|G-X|>0$, there is a vertex $v \in A$. Without loss of generality, $v=\{1, \ldots, k\}$. Each $w \in B$ is not adjacent to $v$, and so $w$ and $v$ intersect. Thus each $w$ must contain at least one of $1, \ldots, k$. Hence at least one of these elements appears in at least $\frac{1}{k}|B|$ of the vertices of $B$, as required.

Without loss of generality, $\left|B_{n}\right| \geqslant \frac{1}{k}|B|$.

Claim 2. $\left|B_{n}\right|>\left(\begin{array}{l}n-3 \\ k-2\end{array}\right)+\left(\begin{array}{l}n-2 \\ k-2\end{array}\right)$.

Proof. $|B| \geqslant \frac{1}{2}|G-X| \geqslant \frac{1}{2}\left(\begin{array}{c}n-1 \\ k-1\end{array}\right)$. Then by Claim 1 and our subsequent assumption, $\left|B_{n}\right| \geqslant \frac{1}{k}|B| \geqslant \frac{1}{2 k}|G-X| \geqslant \frac{1}{2 k}\left(\begin{array}{c}n-1 \\ k-1\end{array}\right)$. Assume for the sake of a contradiction that $\left|B_{n}\right| \leqslant\left(\begin{array}{l}n-3 \\ k-2\end{array}\right)+\left(\begin{array}{l}n-2 \\ k-2\end{array}\right)$. So

$$
\frac{1}{2 k}\left(\begin{array}{c}
n-1 \\
k-1
\end{array}\right) \leqslant\left(\begin{array}{c}
n-3 \\
k-2
\end{array}\right)+\left(\begin{array}{c}
n-2 \\
k-2
\end{array}\right)
$$

Thus

$$
(n-1) ! \leqslant 2 k(k-1)((n-k)(n-3) !+(n-2) !) .
$$

Hence

$$
n^{2}-3 n+2=(n-1)(n-2) \leqslant 2 k(k-1)(2 n-k-2)=4 k^{2} n-4 k n-2 k^{3}-2 k^{2}+4 k .
$$

So $n^{2}+\left(4 k-4 k^{2}-3\right) n+2 k^{3}+2 k^{2}-4 k+2 \leqslant 0$. Since $n \geqslant 4 k^{2}-4 k+3$, it follows $2 k^{3}+2 k^{2}-4 k+2 \leqslant 0$. Given that $k \geqslant 1$, this provides our desired contradiction.

Consider the set $\overline{A_{-n}}$, that is, the complements of the vertices in $A$ that do not contain $n$. So every set in $\overline{A_{-n}}$ contains $n$. Let ${\overline{A_{-n}}}^{*}:=\left\{\bar{v}-n: \bar{v} \in \overline{A_{-n}}\right\}$. That is, remove $n$ from each set in $\overline{A_{-n}}$. There is clearly a one-to-one correspondence between $(n-k)$-sets in $\overline{A_{-n}}$ and $(n-k-1)$-sets in ${\overline{A_{-n}}}^{*}$.

Similarly, define $B_{n}^{*}:=\left\{v-n: v \in B_{n}\right\}$. That is, remove from each vertex of $B_{n}$ the element $n$, which they all contain. The resultant sets are $(k-1)$-sets in $[n-1]$. 
Claim 3. If $v^{*} \in B_{n}^{*}$ and $\bar{w}^{*} \in{\overline{A_{-n}}}^{*}$, then $v^{*} \nsubseteq \bar{w}^{*}$.

Proof. Assume, for the sake of a contradiction, that $v^{*} \subseteq \bar{w}^{*}$. Then it follows that $v \subset \bar{w}$, by re-adding $n$ to both sets. Thus $v$ and $w$ are adjacent. However, $v \in B_{n} \subset B$ and $w \in A_{n} \subset A$, which is a contradiction.

Let $S$ be the $(k-1)$-shadow of ${\overline{A_{-n}}}^{*}$. Hence if $v \in B_{n}^{*}$, then $v \notin S$, by Claim 3. So, it follows that

$$
B_{n}^{*} \subseteq\left(\begin{array}{c}
{[n-1]} \\
k-1
\end{array}\right)-S
$$

Hence we have an upper bound for $\left|B_{n}^{*}\right|$ when we take $|S|$ to be minimised. By Lemma 8, $|S|$ is minimised when ${\overline{A_{-n}}}^{*}$ is first.

Claim 4. $\left|A_{-n}\right| \leqslant\left(\begin{array}{l}n-3 \\ k-2\end{array}\right)$.

Proof. $\left|A_{-n}\right|=\left|\overline{A_{-n}}\right|=\left|{\overline{A_{-n}}}^{*}\right|$, so it is sufficient to show that $\left|{\overline{A_{-n}}}^{*}\right| \leqslant\left(\begin{array}{l}n-3 \\ k-2\end{array}\right)$. Assume for the sake of contradiction that $\left|{\overline{A_{-n}}}^{*}\right| \geqslant\left(\begin{array}{c}n-3 \\ k-2\end{array}\right)=\left(\begin{array}{c}n-3 \\ n-k-1\end{array}\right)$.

Firstly, we show that $|S| \geqslant\left(\begin{array}{l}n-3 \\ k-1\end{array}\right)$. It is sufficient to prove this lower bound when $|S|$ is minimised. Hence we can assume that ${\overline{A_{-n}}}^{*}$ is first, and contains the first $\left(\begin{array}{c}n-3 \\ n-k-1\end{array}\right)$ $(n-k-1)$-sets in the colexicographic ordering. That is, it contains all $(n-k-1)$-sets on $[n-3]$. This is because there are $\left(\begin{array}{c}n-3 \\ n-k-1\end{array}\right)$ such sets, and they come before all other sets in the ordering. In that case, $S$ contains all $(k-1)$-sets in $[n-3]$. Since all of the $(k-1)$-sets in $[n-3]$ are in $S$, it follows that $|S| \geqslant\left(\begin{array}{l}n-3 \\ k-1\end{array}\right)$, as required.

Then it follows that $\left|B_{n}^{*}\right| \leqslant\left(\begin{array}{l}n-1 \\ k-1\end{array}\right)-\left(\begin{array}{l}n-3 \\ k-1\end{array}\right)=\left(\begin{array}{l}n-3 \\ k-2\end{array}\right)+\left(\begin{array}{l}n-2 \\ k-2\end{array}\right)$. However, $\left|B_{n}^{*}\right|=\left|B_{n}\right|>$ $\left(\begin{array}{l}n-3 \\ k-2\end{array}\right)+\left(\begin{array}{l}n-2 \\ k-2\end{array}\right)$ by Claim 2. This provides our desired contradiction.

Claim 5. $\left|A_{n}\right| \geqslant \frac{k}{k+1}|A|$.

Proof. First we show that $\left|A_{n}\right| \geqslant k\left|A_{-n}\right|$. Suppose otherwise, for the sake of a contradiction. By Claim 4, $|A|=\left|A_{n}\right|+\left|A_{-n}\right|<(k+1)\left|A_{-n}\right| \leqslant(k+1)\left(\begin{array}{l}n-3 \\ k-2\end{array}\right)$. But $|A| \geqslant(1-p)|G-X|$. Hence $(1-p)\left(\begin{array}{l}n-1 \\ k-1\end{array}\right)<(k+1)\left(\begin{array}{l}n-3 \\ k-2\end{array}\right)$. Thus $(n-1)(n-2)<$ $\frac{1}{1-p}(k+1)(k-1)(n-k) \leqslant \frac{1}{1-p}(k+1)(k-1)(n-2)$. Thus $n<\frac{1}{1-p}\left(k^{2}-1\right)+1$, which contradicts our lower bound on $n$.

Then $\left|A_{n}\right| \geqslant k\left|A_{-n}\right|=k\left(|A|-\left|A_{n}\right|\right)$. So $(k+1)\left|A_{n}\right| \geqslant k|A|$ as required.

Claim 6. $B_{n}=B$.

Proof. Suppose, for the sake of a contradiction, that there exists some vertex $v \in B$ such that $n \notin v$. So each $w \in A_{n}$ contains $n$ (by definition) and some element of $v$ (which is not $n$ ), since $v w$ is not an edge. Any vertex of $A_{n}$ can be constructed as follows - take element $n$, choose one of the $k$ elements of $v$, and choose the remaining $k-2$ elements from the remaining $n-2$ elements of $[n]$. Thus

$$
\left|A_{n}\right| \leqslant 1 \cdot k\left(\begin{array}{l}
n-2 \\
k-2
\end{array}\right)
$$


Note this is actually a weak upper bound, since we have counted some of the vertices of $A_{n}$ more than once. Recall $|A| \geqslant(1-p)|G-X| \geqslant(1-p)\left(\begin{array}{l}n-1 \\ k-1\end{array}\right)$. So by Claim 5 ,

$$
\frac{(1-p) k}{(k+1)}\left(\begin{array}{l}
n-1 \\
k-1
\end{array}\right) \leqslant \frac{k}{k+1}|A| \leqslant k\left(\begin{array}{l}
n-2 \\
k-2
\end{array}\right) .
$$

Thus $\frac{n-1}{k-1} \leqslant \frac{1}{1-p}(k+1)$ and $n \leqslant \frac{1}{1-p}\left(k^{2}-1\right)+1$, which contradicts our lower bound on $n$.

Claim 7. $A_{n}=A$.

Proof. This follows by essentially the same argument as Claim 6. Assume our claim does not hold and there exists $v \in A$ such that $n \notin v$. By Claim $6,\left|B_{n}\right|=|B| \geqslant \frac{1}{2}\left(\begin{array}{l}n-1 \\ k-1\end{array}\right)$. There is an upper bound on $\left|B_{n}\right|$ equal to the upper bound on $\left|A_{n}\right|$ in the previous proof. Then

$$
\frac{1}{2}\left(\begin{array}{l}
n-1 \\
k-1
\end{array}\right) \leqslant|B|=\left|B_{n}\right| \leqslant k\left(\begin{array}{l}
n-2 \\
k-2
\end{array}\right),
$$

and so $n \leqslant 2 k(k-1)+1$. This contradicts our lower bound on $n$.

Claims 6 and 7 show that every vertex in $G-X=A \cup B$ contains $n$. Thus $|G-X| \leqslant$ $\left(\begin{array}{l}n-1 \\ k-1\end{array}\right)$ and $|X| \geqslant\left(\begin{array}{c}n-1 \\ k\end{array}\right)$, our desired contradiction.

By Lemma 11, if $X$ is a $\frac{2}{3}$-separator of the Kneser graph $G$ and $n \geqslant 4 k^{2}-4 k+3$, then $|X| \geqslant\left(\begin{array}{c}n-1 \\ k\end{array}\right)$. Hence by Theorem $4, \operatorname{tw}(G) \geqslant\left(\begin{array}{c}n-1 \\ k\end{array}\right)-1$. This proves Theorem 1 .

Also, Lemma 11 allows us to prove Theorem 3.

Proof of Theorem 3. Let $C_{1}, \ldots, C_{r}$ be the colour classes of $H$. Recall $G=\operatorname{Kneser}(n, k)$. Let $X:=V(\bar{G})-V(H)$, so that $X, C_{1}, \ldots, C_{r}$ is a partition of the vertex set of $\bar{G}$ (and also $G$ ). In $G$ there are no edges between any pair $C_{i}, C_{j}$, and $\left|C_{i}\right| \leqslant p|H|=p|G-X|$ for each $i$. So $X$ is a $p$-separator of $G$, and $|X| \geqslant\left(\begin{array}{c}n-1 \\ k\end{array}\right)$ by Lemma 11 . Hence $|H| \leqslant\left(\begin{array}{l}n-1 \\ k-1\end{array}\right)$.

\section{Lower Bound for Treewidth in Theorem 2}

To complete our proof of Theorem 2, we need to obtain a lower bound on the treewidth when $k=2$. If $n \leqslant 4$, then Theorem 2 is trivial. When $n=5$, then $G$ is the Petersen graph, which contains a $K_{5}$-minor forcing $\operatorname{tw}(G) \geqslant 4$. Hence we may assume that $n \geqslant 6$.

Assume, for the sake of a contradiction that $\operatorname{tw}(G)<\left(\begin{array}{c}n-1 \\ 2\end{array}\right)-1$. Let $\left(T,\left(B_{x}: x \in\right.\right.$ $V(T))$ ) be a minimum width tree decomposition for $G$, and normalise the tree decomposition such that if $x y \in E(T)$, then $B_{x} \nsubseteq B_{y}$ and $B_{y} \nsubseteq B_{x}$. By Theorem 4 , there exists a $\frac{2}{3}$-separator $X$ such that $|X|<\left(\begin{array}{c}n-1 \\ 2\end{array}\right)$. In fact, by the original proof in [16], we can go further and assert that $X$ is a subset of a bag of $\left(B_{x}: x \in V(T)\right)$.

Now $|G-X|=\left(\begin{array}{l}n \\ 2\end{array}\right)-|X|>\left(\begin{array}{c}n-1 \\ 1\end{array}\right)=n-1$. By Lemma $5, V(G-X)$ has two parts $A$ and $B$ such that $\frac{1}{3}|G-X| \leqslant|A|,|B| \leqslant \frac{2}{3}|G-X|$ and there is no edge with an endpoint in $A$ and $B$. (Note that this bound on $|A|$ and $|B|$ is slightly weaker than in Lemma 5 , but 
has the benefit of being the same on both parts.) Since $n \geqslant 6$, it follows that $|A|,|B| \geqslant 2$. By Theorem $6, V(G-X)$ is too large to be an independent set, and so it contains an edge, with both endpoints in $A$ or both endpoints in $B$.

Without loss of generality this edge is $\{1,2\}\{3,4\} \in A$. Then $B \subseteq\{\{1,3\},\{1,4\}$, $\{2,3\},\{2,4\}\}$. If $B$ contains an edge, then $V(G-X) \subseteq\{\{1,2\},\{1,3\},\{1,4\},\{2,3\}$, $\{2,4\},\{3,4\}\}$ and has maximum order 6 . Otherwise, without loss of generality, $B=$ $\{\{1,3\},\{1,4\}\}$ and $A=\{\{3,4\},\{1, i\} \mid i \notin\{1,3,4\}\}$, so $|G-X|=n$. (Note $A$ must be exactly that set, or $|G-X|$ is too small.)

If $n \geqslant 7$, then $|G-X| \geqslant 7$ and the first case cannot occur. However in the second case, $|B|=2<\frac{1}{3} \cdot 7 \leqslant \frac{1}{3} n$. So neither case can occur, and we have forced a contradiction on either $|G-X|$ or $|B|$. This completes the proof when $n \geqslant 7$. Hence, let $n=6$, and note $|G-X|=6$ in either case.

Now we use the fact that $X$ is a subset of some bag $B_{x}$. Now for all $x \in V(T)$, $\left|B_{x}\right| \leqslant\left(\begin{array}{l}5 \\ 2\end{array}\right)-1=9$. Since $|G-X|=6$, it follows $|X|=9$. Hence $X$ is exactly a bag of maximum order. For either choice of $G-X$, note that $A$ is a connected component. So there is some subtree of $T-x$ that contains all vertices of $A$. Let $y$ be the node of this subtree adjacent to $x$. Also note, for either choice of $G-X$, that each vertex of $X$ has a neighbour in $A$. So every vertex of $B_{x}$ is also in bag $B_{y}$, which contradicts our normalisation.

Thus, if $n \geqslant 6$, then $\operatorname{tw}(G) \geqslant\left(\begin{array}{c}n-1 \\ 2\end{array}\right)-1$. This completes the proof of Theorem 2 .

\section{Open Questions}

We conjecture that Theorem 1 should also hold for smaller values of $n$.

Conjecture 12. Let $G$ be a Kneser graph with $n \geqslant 3 k$ and $k \geqslant 2$. Then $\operatorname{tw}(G)=$ $\left(\begin{array}{c}n-1 \\ k\end{array}\right)-1$.

This conjecture follows directly from Theorem 2 when $k=2$. The Petersen graph also shows that $n \geqslant 3 k$ is a tight bound when $k=2$.

In general, we can determine a slightly better tree decomposition when $n<3 k-1$. Let $X=\{v \in V(G): 1 \in v\}$, and let $W$ be an independent set in $V(G)-X$ such that no two vertices of $W$ have a common neighbour in $X$. We define a tree decomposition for $G$ with underlying tree $T$ as follows. Let $r$ denote the root node of $T$, and let $r$ have one child node for each vertex in $W$ and each vertex in $X$ adjacent to no vertex in $W$. Label each of these child nodes by their associated vertex of $G$. Let each node labeled by a vertex $w \in W$ have one child node for each vertex of $N(w) \cap X$. Label each of those child nodes by their associated vertex of $G$, and note that since every vertex of $X$ has at most one neighbour in $W$, no vertex of $G$ labels more than one node of $T$.

Define the bag indexed by $r$ to be $V(G)-W-X$. Note this bag contains less than $\left(\begin{array}{c}n-1 \\ k\end{array}\right)$ vertices when $W \neq \emptyset$. If a node is labeled by a vertex $v \in X$, let the corresponding bag be $N(v) \cup\{v\}$. These bags contain $\left(\begin{array}{c}n-k \\ k\end{array}\right)+1$ vertices. If a node is labeled by a vertex $w \in W$, let the corresponding bag be $\{w\} \cup\{u: u w \in E(G), 1 \notin u\} \cup\{u: u x \in E(G)$ 
where $x w \in E(G)$ and $1 \in x\}$. These bags contain less than $\left(\begin{array}{c}n-1 \\ k\end{array}\right)$ vertices whenever $|W| \geqslant 2$, since they contain no vertex in $X$, and each contains only one vertex from $W$. This is a valid tree decomposition, but we omit the proof. When $|W| \geqslant 2$, the width of this tree decomposition is less than the width given by Lemma 9 .

However, when $|W| \leqslant 1$, this tree decomposition has the same width as given by Lemma 9. We can construct $W$ such that $|W| \geqslant 2$ iff $n<3 k-1$. For example, let $W=\{\{2, \ldots,(k+1)\},\{(k+1), \ldots, 2 k\}\}$. If $n \leqslant 3 k-2$, then any vertex of $X$ must be non-adjacent to at least one vertex of $W$. Alternatively, if $n \geqslant 3 k-1$ and $|W| \geqslant 2$, then there exists two vertices $x, y \in W$ such that $|x \cup y| \leqslant 2 k-1$. Then $X$ contains a vertex adjacent to both $x$ and $y$. Hence, for general $n$, we cannot improve the lower bound on $n$ in Theorem 1 to $3 k-2$ or below. This does leave a question about what may occur for $n=3 k-1$. It is possible that Theorem 1 holds for $n \geqslant 3 k-1$, with the Petersen graph as a single exception.

\section{Acknowledgements}

Thanks to Alex Scott for helpful conversations, and for pointing out references $[4,5,18]$.

\section{References}

[1] Hans L. Bodlaender. A tourist guide through treewidth. Acta Cybernet., 11(1-2):121, 1993.

[2] Paul Erdős, Chao Ko, and Richard Rado. Intersection theorems for systems of finite sets. Quart. J. Math. Oxford Ser. (2), 12:313-320, 1961.

[3] Péter Frankl. A new short proof for the Kruskal-Katona theorem. Discrete Math., 48(2-3):327-329, 1984.

[4] Dániel Gerbner, Nathan Lemons, Cory Palmer, Dömötör Pálvölgyi, Balázs Patkós, and Vajk Szécsi. Almost cross-intersecting and almost cross-sperner pairs of families of sets. Graphs and Combinatorics, 29:489-498, 2013.

[5] Dániel Gerbner, Nathan Lemons, Cory Palmer, Balázs Patkós, and Vajk Szécsi. Almost intersecting families of sets. SIAM. Discrete Mathematics, 26(4):1657-1669, 2012 .

[6] Rudolf Halin. S-functions for graphs. J. Geometry, 8(1-2):171-186, 1976.

[7] Gyula O. H. Katona. A simple proof of the Erdős-Chao Ko-Rado theorem. J. Combin. Theory Ser. B, 13:183-184, 1972.

[8] Gyula O.H. Katona. A theorem of finite sets. In Theory of graphs (Proc. Colloq., Tihany, 1966), pages 187-207. Academic Press, New York, 1968.

[9] Martin Kneser. Aufgabe 360. Jahresber. Deutsch. Math.-Verein., 58:27, 1955.

[10] Joseph B. Kruskal. The number of simplices in a complex. In Mathematical optimization techniques, pages 251-278. Univ. of California Press, Berkeley, Calif., 1963. 
[11] László Lovász. Kneser's conjecture, chromatic number, and homotopy. J. Combin. Theory Ser. A, 25(3):319-324, 1978.

[12] Lambertus Marchal. Treewidth. PhD thesis, Maastricht University, 2012.

[13] Makoto Matsumoto and Norihide Tokushige. The exact bound in the Erdős-Ko-Rado theorem for cross-intersecting families. J. Combin. Theory Ser. A, 52(1):90-97, 1989.

[14] László Pyber. A new generalization of the Erdős-Ko-Rado theorem. J. Combin. Theory Ser. A, 43(1):85-90, 1986.

[15] Neil Robertson and Paul D. Seymour. Graph minors I-XXIII. J. Combin. Theory Ser. B, 1983-2012.

[16] Neil Robertson and Paul D. Seymour. Graph minors. II. Algorithmic aspects of tree-width. J. Algorithms, 7(3):309-322, 1986.

[17] Edward R. Scheinerman and Daniel H. Ullman. Fractional graph theory. Wiley, 1997.

[18] Alex Scott and Elizabeth Wilmer. Hypergraphs of bounded disjointness. 2013. arXiv: 1306.4236.

[19] Günter M. Ziegler. Generalized Kneser coloring theorems with combinatorial proofs. Inventiones Math, 147:671-691, 2001. 